\title{
SPACE-TIME FINITE ELEMENT METHODS FOR SECOND-ORDER HYPERBOLIC EQUATIONS*
}

\author{
Gregory M. HULBERT \\ Department of Mechanical Engineering and Applied Mechanics, 321 W.E. Lay Automotive Lab, N.C., \\ The University of Michigan, Ann Arbor, MI 48109-2121, U.S.A. \\ Thomas J.R. HUGHES \\ Institute for Computer Methods in Applied Mechanics and Engineering, Division of Applied Mechanics, \\ Durand Building, Stanford University, Stanford, CA 94305, U.S.A.
}

Received March 1990

\begin{abstract}
Space-time finite element methods are presented to accurately solve elastodynamics problems that include sharp gradients due to propagating waves. The new methodology involves finite element discretization of the time domain as well as the usual finite element discretization of the spatial domain. Linear stabilizing mechanisms are included which do not degrade the accuracy of the space-time finite element formulation. Nonlinear discontinuity-capturing operators are used which result in more accurate capturing of steep fronts in transient solutions while maintaining the high-order accuracy of the underlying linear algorithm in smooth regions. The space-time finite element method possesses a firm mathematical foundation in that stability and convergence of the method have been proved. In addition, the formulation has been extended to structural dynamics problems and can be extended to higher-order hyperbolic systems.
\end{abstract}

\section{Introduction}

While finite element methods have been widely used to solve time-dependent problems, most procedures have been based upon semidiscretizations: the spatial domain is discretized using finite elements, producing a system of ordinary differential equations in time which in turn is discretized using finite difference methods for ordinary differential equations. These procedures have been particularly successful in solving structural dynamics problems. (Structural dynamics problems typically generate a stiff system of ordinary differential equations; the low frequency response of the system is of principal interest.) One disadvantage of the semidiscrete approach is the difficulty in designing algorithms that accurately capture discontinuities or sharp gradients in the solution. To illustrate this difficulty, we consider the impact against a rigid wall of a one-dimensional, homogeneous elastic bar; see Fig. 1. Initially, the bar is moving with a uniform speed; the left end of the bar then impacts the rigid wall. This generates a compressive stress wave in the bar that starts at the impacted end and propagates

* This research was sponsored by the U.S. Office of Naval Research under Contract Number N00014-84-K-0600. 

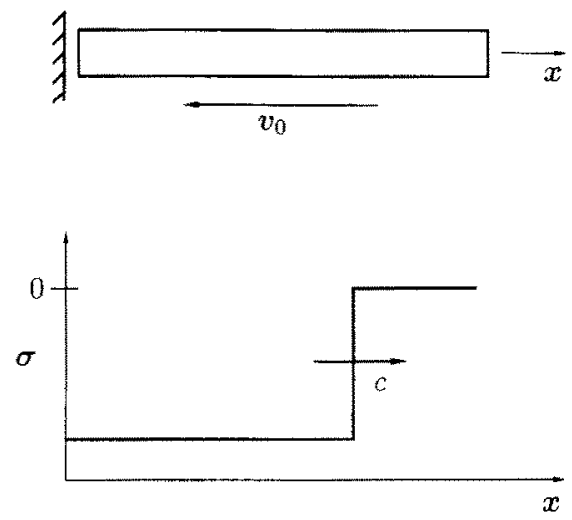

Fig. 1. One-dimensional elastic bar impact problem. (Top: model problem; bottom: exact solution.)

towards the free end. Attention is restricted to the time interval during which the stress wave remains compressive, that is, before the stress wave reaches the free end of the bar. The bar has a length of 4; the density, area and Young's modulus have unit values; the uniform initial speed also has unit value. The bar was uniformly discretized using 200 quadratic rod elements. Figure 2 shows the stress distribution in the bar for time $t=2.81$, calculated using the trapezoidal rule algorithm with time step $\Delta t=0.01$; the dotted line denotes the exact solution. The oscillations in the trapezoidal rule solution induced by the discontinuity are clearly evident. These oscillations are not surprising as it is well-known that the trapezoidal rule algorithm possesses no numerical damping to localize or limit oscillations.

Within the context of structural dynamics, many semidiscrete algorithms have been developed which possess numerical damping but still retain the accuracy characteristics of the trapezoidal rule algorithm for problems with smooth solutions. One of the more successful semidiscrete algorithms is the Hilber-Hughes-Taylor $\alpha$ method (HHT- $\alpha$ method) [1-5]. The stress distribution in the bar is shown in Fig. 3 calculated using the HHT- $\alpha$ algorithm with

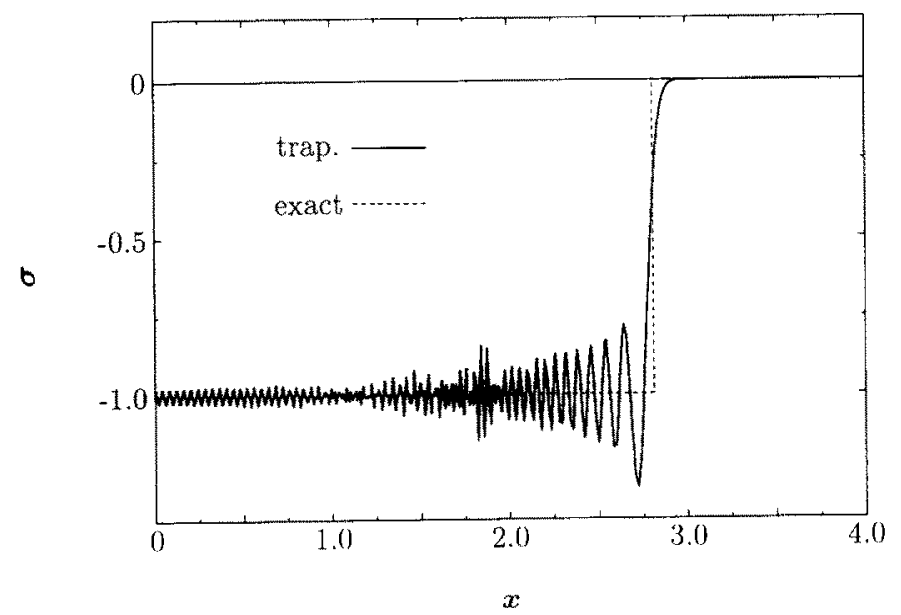

Fig. 2. Bar impact problem. Stress distribution calculated using trapezoidal rule algorithm. 


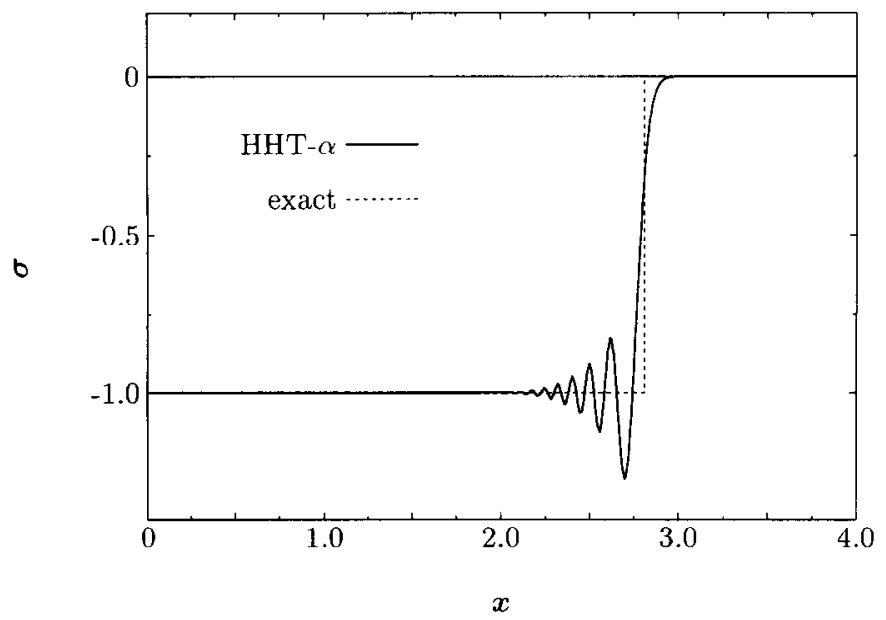

Fig. 3. Bar impact problem. Stress distribution calculated using Hilber-Hughes-Taylor $\alpha$ algorithm.

$\alpha=-0.3$ and $\Delta t=0.01(t=2.81)$. Compared to the trapezoidal rule algorithm, the HHT- $\alpha$ method significantly localizes oscillations although there is still a number of oscillations near the discontinuity.

These results are indicative of the performance of structural dynamics algorithms when solving wave propagation problems. While these semidiscrete methods are effective for computing smooth responses (low frequency response) of interest in structural dynamics, their performance is less satisfactory when solving problems exhibiting discontinuities or sharp gradients in their solutions.

Another disadvantage of semidiscrete methods may be seen using a second example problem. Consider a one-dimensional elastic bar consisting of two different materials; as shown in Fig. 4, the elastic wave speed in the first material is greater than that of the second material. At the initial time, non-uniform traction is applied to the bar near the material interface. Of interest in this problem is tracking the relatively sharp stress distribution as it propagates throughout the bar. Since, for semidiscrete methods, the spatial domain is discretized first and then the same temporal discretization is used for the resultant set of ordinary differential equations, the corresponding space-time discretization is structured. That

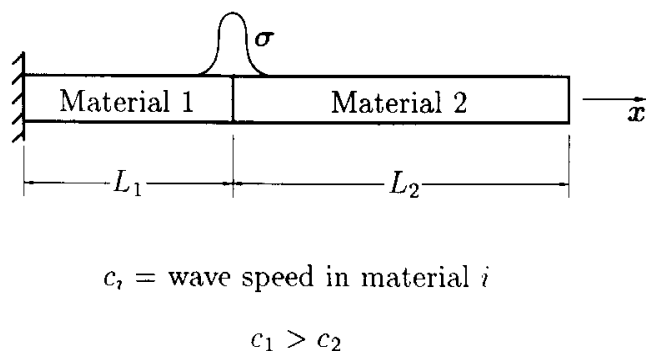

Fig. 4. Two-material elastic bar problem. 
is, as shown in Fig. 5, the space-time discretization arising from the semidiscrete approach consists of rectangular subdomains of the space-time domain. In contrast, space-time finite element methods, in which the spatial and temporal domains are simultaneously discretized, accomodate unstructured meshes in the space-time domain, such as the mesh shown in Fig. 6. This mesh may be considered to arise from an adaptive mesh refinement strategy in which both spatial and temporal refinement can occur to accurately capture the stress waves as they propagate throughout the bar. In regions where the solution is smooth, the mesh is relatively coarse while a finer mesh is employed near the stress wave fronts. Thus, an accurate solution may be obtained without resorting to a uniformly-refined (and computationally expensive) mesh. Adaptive mesh refinement strategies using space-time finite element methods recently have been developed by Johnson and co-workers for fluid dynamics problems [6].

The use of finite elements to discretize the temporal domain as well as the spatial domain was first proposed by Argyris and Scharpf [7], Fried [8] and Oden [9]. The underlying concept of their formulations is the application of Hamilton's principle for dynamics. Numerous research efforts ensued based upon variants of Hamilton's principle and Hamilton's law for dynamics and elastodynamics, and a variational principle for heat conduction first presented by Gurtin [10]. See, e.g., [11-24]. (This is not meant to be an exhaustive list; the interested reader is encouraged to study the reference lists of the above papers. Good compendia of space-time finite element formulations based on Hamilton's principle and Hamilton's law may be found in $[13,25]$.)

A second approach towards formulating space-time finite element methods is to work directly with the differential equations or variational formulation rather than from a variational principle. Numerous problems, including elastodynamics, heat conduction and advectivediffusive systems associated with fluid dynamics, have been solved using space-time finite element methods in which the unknown quantities were assumed to be continuous with respect to time. See [25-43] for examples of time-continuous Galerkin formulations. Also,

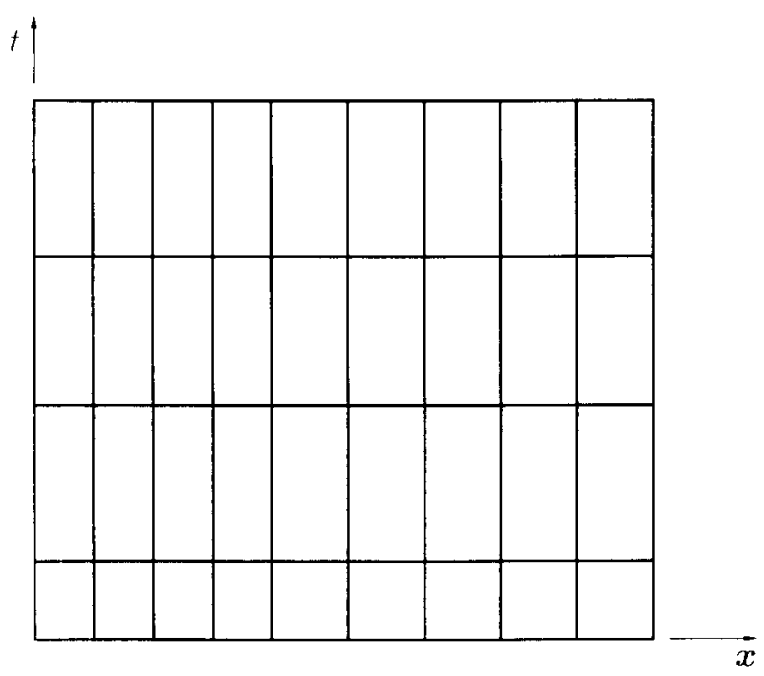

Fig. 5. Semidiscrete space-time mesh for the twomaterial elastic bar problem.

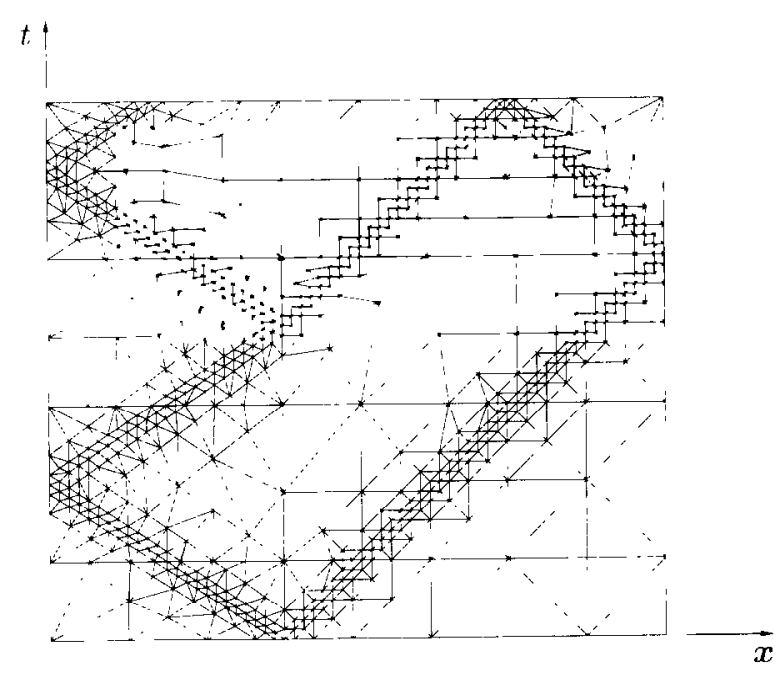

Fig. 6. Space-time finite element mesh for the twomaterial elastic bar problem. 
using continuous functions in time, the ordinary differential equations emanating from semidiscretizations were multiplied by weighting functions and integrated over time intervals; see [44-49]. Many traditional ordinary differential equation algorithms were rederived in this manner.

Another approach, working from the differential equation viewpoint, has evolved during the last fifteen years. The idea is to permit the unknown fields to be discontinuous with respect to time. The time-discontinuous Galerkin method was originally developed for first-order hyperbolic equations [50,51]. This method has been successfully applied to problems in incompressible and compressible fluid dynamics and heat conduction; see [52-64].

The time-discontinuous Galerkin method leads to stable, higher-order accurate finite element methods. It was first shown in $[51,53,65]$ that the time-discontinuous Galerkin method leads to $A$-stable, higher-order accurate ordinary diffential equation solvers. This is in contrast to the conditional stability of some time-continuous Galerkin methods observed by Bajer [26, 27] and Howard and Penny [15]. Furthermore, the time-discontinuous framework seems conducive to the establishment of rigorous convergence proofs and error estimates $[6,54,55,57,58,62,65-73]$.

Based on the success of the time-discontinuous Galerkin method for first-order systems, it is desirable to extend the method to problems involving second-order hyperbolic equations, e.g., elastodynamics. Classical elastodynamics can be converted to first-order symmetric hyperbolic form, which has proved useful in theoretical studies [74]. Finite element methods for first-order symmetric hyperbolic systems are thus immediately applicable [54, 55, 57]. However, there are disadvantages to this approach: in symmetric hyperbolic form the state vector consists of displacements, velocities and stresses, which is computationally uneconomical; and the generalization to nonlinear elastodynamics seems possible only in special circumstances [75].

In [76], we developed space-time finite element methods for elastodynamics based on the natural framework of second-order hyperbolic equations. Independent interpolations were permitted for the displacement and velocity fields; error estimates were derived and numerical results demonstrated the good performance of the new methods. In this paper, a 'single-field' formulation is employed in which the displacement field is interpolated; nonlinear operators are then introduced to control the oscillations induced by discontinuous solutions.

To date, we have concentrated on developing space-time finite element methods for elastodynamics and time finite element methods for the ordinary differential equations associated with structural dynamics. These equations are prototypes of general second-order hyperbolic systems; consequently, the methods developed have wider applicability than structural dynamics and elastodynamics. In this paper, attention is restricted to linear elastodynamics.

An outline of the paper follows. A brief review of the equations of linear elastodynamics is given in Section 2. The space-time finite element method is presented in Section 3; the proposed formulation consists of several essential facets. Appended to the time-discontinuous Galerkin formulation are stabilizing operators that have least-squares form. (Similar stabilization ideas have been exploited for other problems by Hughes et al. [77-81], Franca and Hughes [82] and Loula et al. [83-85].) Nonlinear discontinuity-capturing operators are added to improve the performance of the algorithm in regions where the solution cxhibits sharp gradients. (Within the context of finite element formulations for fluid mechanics, discon- 
tinuity-capturing operators have been developed by Hughes et al. [86], Hughes and Mallet [87], Johnson and Szepessy [70,71, 88], Szepessy [73], Galeão and Dutra do Carmo [89, 90] and Shakib [63].) The importance of each component of the formulation is emphasized by comparing numerical results obtained for the elastic bar impact problem; these results also demonstrate the improved performance of the space-time finite element method for wave propagation problems when compared to typical semidiscrete methods. In Section 4, results are presented from stability and convergence analyses of the space-time finite element methods. Finally, conclusions are drawn in Section 5.

\section{Classical linear elastodynamics}

Consider an elastic body occupying an open, bounded region $\Omega \subset \mathbb{R}^{d}$, where $d$ is the number of space dimensions. The boundary of $\Omega$ is denoted by $\Gamma$. Let $\Gamma_{g}$ and $\Gamma_{h}$ denote non-overlapping subregions of $\Gamma$ such that

$$
\Gamma=\overline{\Gamma_{\mathrm{g}} \cup \Gamma_{h}} .
$$

The displacement vector is denoted by $u(x, t)$, where $x \in \bar{\Omega}$ and $t \in[0, T]$, the time interval of length $T>0$. The stress is determined by the generalized Hooke's law:

$$
\boldsymbol{\sigma}(\nabla u)=\boldsymbol{c} \cdot \nabla \boldsymbol{u}
$$

or, in components,

$$
\sigma_{i j}=c_{i j k l} u_{k, l},
$$

where $1 \leqslant i, j, k, l \leqslant d, u_{k, l}=\partial u_{k} / \partial x_{l}$, and summation over repeated indices is implied. The elastic coefficients $c_{i j k l}=c_{i j k l}(x)$ are assumed to satisfy the following conditions:

$$
\begin{array}{ll}
c_{i j k l}=c_{j i k l}=c_{i j l k} & \text { (minor symmetries), } \\
c_{i j k l}=c_{k l i j} & \text { (major symmetry), } \\
c_{i j k l} \psi_{i j} \psi_{k l}>0 \quad \forall \psi_{i j}=\psi_{j i} \neq 0 & \text { (positive definiteness). }
\end{array}
$$

By the minor symmetries, $\sigma$ is symmetric and depends only upon the symmetric part of $\nabla \boldsymbol{u}$. The minor symmetries play no role in the formulation presented in subsequent sections; however, the major symmetry and positive definiteness are used in the stability and convergence proofs.

The equations of the initial/boundary-value problem of elastodynamics are

$$
\begin{aligned}
& \rho \ddot{u}=\nabla \cdot \sigma(\nabla u)+f \quad \text { on } Q \equiv \Omega \times] 0, T[, \\
& \left.u=g \quad \text { on } Y_{g} \equiv \Gamma_{g} \times\right] 0, T[,
\end{aligned}
$$




$$
\begin{aligned}
& \left.\boldsymbol{n} \cdot \boldsymbol{\sigma}(\nabla u)=\boldsymbol{h} \text { on } Y_{h} \equiv \Gamma_{h} \times\right] 0, T[ \\
& u(x, 0)=u_{0}(x) \text { for } x \in \Omega \\
& \dot{u}(x, 0)=v_{0}(x) \text { for } x \in \Omega,
\end{aligned}
$$

where $\rho=\rho(x)>0$ is the density, a superposed dot indicates partial differentiation with respect to $t, f$ is the body force, $g$ is the prescribed boundary displacement, $h$ is the prescribed boundary traction, $\boldsymbol{u}_{0}$ is the initial displacement, $\boldsymbol{v}_{0}$ is the initial velocity and $n$ is the unit outward normal to $\Gamma$. In components, $\nabla \cdot \sigma$ and $n \cdot \sigma$ are $\sigma_{i j, j}$ and $\sigma_{i j} n_{j}$, respectively. The objective is to find a $\boldsymbol{u}$ that satisfies (7)-(11) for given $\rho, \boldsymbol{c}, \boldsymbol{f}, \boldsymbol{g}, \boldsymbol{h}, \boldsymbol{u}_{0}$ and $\boldsymbol{v}_{0}$.

REMARK. The form of the generalized Hooke's law, (2), was chosen as it enables a generalization of the proposed finite element formulation to the nonlinear elastodynamics case where the first Piola-Kirchhoff stress, $\boldsymbol{P}$, replaces $\boldsymbol{\sigma}$; see [91].

\section{A space-time Galerkin/least-squares finite element formulation}

\subsection{Preliminaries}

Consider a partition of the time domain, $I=] 0, T$, having the form: $0=t_{0}<t_{1}<\cdots<$ $t_{N}=T$. Let $\left.I_{n}=\right] t_{n-1}, t_{n}\left[\right.$ and $\Delta t_{n}=t_{n}-t_{n-1}$. Referring to Fig. 7 , the following notations are employed:
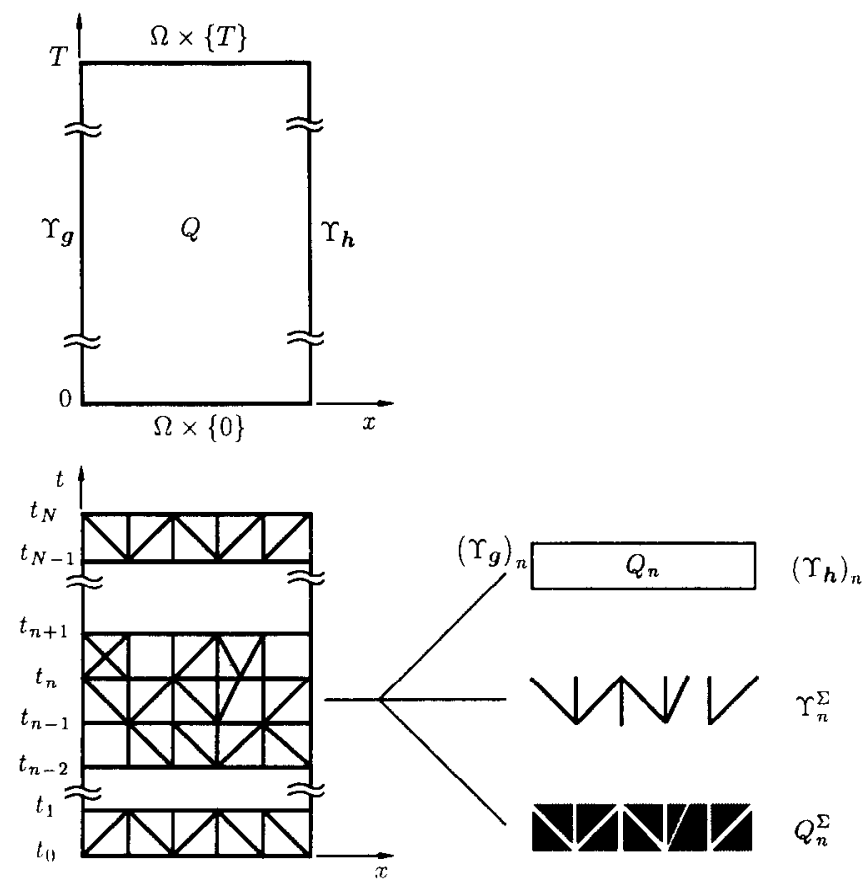

Fig. 7. Illustration of space-time finite element notation. 


$$
\begin{aligned}
& Q_{n}=\Omega \times I_{n}, \\
& Y_{n}=\Gamma \times I_{n}, \\
& \left(Y_{g}\right)_{n}=\Gamma_{g} \times I_{n}, \\
& \left(Y_{h}\right)_{n}=\Gamma_{h} \times I_{n} .
\end{aligned}
$$

$Q_{n}$ is referred to as the $n$th space-time slab.

Let $\left(n_{\mathrm{el}}\right)_{n}$ denote the number of space-time elements in $Q_{n} ; Q_{n}^{e} \subset Q_{n}$ denotes the interior of the $e$ th element; $Y_{n}^{e}$ denotes its boundary. Let

$$
\begin{array}{ll}
Q_{n}^{\Sigma}=\bigcup_{e=1}^{\left(n_{\mathrm{cl}}\right)_{n}} Q_{n}^{e} & \text { (element interiors), } \\
Y_{n}^{\Sigma}=\bigcup_{e=1}^{\left(n_{\mathrm{e} 1}\right)_{n}} Y_{n}^{e}-Y_{n} & \text { (interior boundary). }
\end{array}
$$

Frequently-used notations are

$$
\begin{aligned}
& \left(\boldsymbol{w}^{h}, \boldsymbol{u}^{h}\right)_{\Omega}=\int_{\Omega} \boldsymbol{w}^{h} \cdot \boldsymbol{u}^{h} \mathrm{~d} \Omega, \\
& a\left(\boldsymbol{w}^{h}, \boldsymbol{u}^{h}\right)_{\Omega}=\int_{\Omega} \nabla \boldsymbol{w}^{h} \cdot \boldsymbol{\sigma}\left(\nabla \boldsymbol{u}^{h}\right) \mathrm{d} \Omega, \\
& \left(\boldsymbol{w}^{h}, \boldsymbol{u}^{h}\right)_{Q_{n}}=\int_{Q_{n}} \boldsymbol{w}^{h} \cdot \boldsymbol{u}^{h} \mathrm{~d} Q, \\
& a\left(\boldsymbol{w}^{h}, \boldsymbol{u}^{h}\right)_{Q_{n}}=\int_{Q_{n}} \nabla \boldsymbol{w}^{h} \cdot \boldsymbol{\sigma}\left(\nabla \boldsymbol{u}^{h}\right) \mathrm{d} Q, \\
& \left(\boldsymbol{w}^{h}, \boldsymbol{u}^{h}\right)_{Q_{n}^{\Sigma}}=\int_{Q_{n}^{\Sigma}} \boldsymbol{w}^{h} \cdot \boldsymbol{u}^{h} \mathrm{~d} Q, \\
& \left(\boldsymbol{w}^{h}, \boldsymbol{u}^{h}\right)_{Y_{n}^{\Sigma}}=\int_{Y_{n}^{\Sigma}} \boldsymbol{w}^{h} \cdot \boldsymbol{u}^{h} \mathrm{~d} Y, \\
& \left(\boldsymbol{w}^{h}, \boldsymbol{u}^{h}\right)_{\left(Y_{h}\right)_{n}}=\int_{\left(Y_{h}\right)_{n}} \boldsymbol{w}^{h} \cdot \boldsymbol{u}^{h} \mathrm{~d} Y,
\end{aligned}
$$

where

$$
\begin{aligned}
& \int_{Q_{n}} \cdots \mathrm{d} Q=\int_{I_{n}} \int_{\Omega} \cdots \mathrm{d} \Omega \mathrm{d} t, \\
& \int_{Q_{n}^{\Sigma}} \cdots \mathrm{d} Q=\sum_{e=1}^{\left(n_{\mathrm{el}}\right)_{n}} \int_{Q_{n}^{e}} \cdots \mathrm{d} Q,
\end{aligned}
$$




$$
\int_{\left(Y_{h}\right)_{n}} \cdots \mathrm{d} Y=\int_{I_{n}} \int_{Y_{h}} \cdots \mathrm{d} \Gamma \mathrm{d} t
$$

and $a(\cdot, \cdot)_{\Omega}$ is the strain-energy inner product.

Consider a typical space-time element with domain $Q^{e}$ and boundary $Y^{e}$. Let $n$ denote the outward unit normal vector to $Y^{e} \cap\{t\}$ in the spatial hyperplane $Q^{e} \cap\{t\}$. If $Q^{e}$ equals the product of $\Omega^{e}$ and a time interval, then $\boldsymbol{n}$ is the usual spatial unit outward normal vector to $\Gamma^{e}$.

Assuming the function $w(t)$ to be discontinuous at time $t_{n}$, the temporal jump operator is defined by

where

$$
\llbracket \boldsymbol{w}\left(t_{n}\right) \rrbracket=\boldsymbol{w}\left(t_{n}^{+}\right)-\boldsymbol{w}\left(t_{n}^{-}\right),
$$

$$
w\left(t_{n}^{ \pm}\right)=\lim _{\varepsilon \rightarrow 0 \pm} w\left(t_{n}+\varepsilon\right) .
$$

The argument $\boldsymbol{x}$ has been suppressed in (28), (29) to simplify the notation.

Consider two adjacent space-time elements. Designate one element by + and the other by - ; let $\boldsymbol{n}^{+}$and $\boldsymbol{n}^{-}$denote the spatial unit outward normal vectors along the interface (see Fig. $8)$. To simplify the notation, the argument $t$ is suppressed. Assuming the function $w(x)$ is discontinuous at point $\boldsymbol{x}$, the spatial jump operator is defined by

where

$$
\llbracket w(x) \rrbracket=w\left(x^{+}\right)-w\left(x^{-}\right),
$$

$$
\begin{aligned}
& w\left(x^{ \pm}\right)=\lim _{\varepsilon \rightarrow 0 \pm} w(x+\varepsilon n), \\
& n=n^{+}=-n^{-} .
\end{aligned}
$$

Then

$$
\begin{aligned}
\llbracket \sigma(\nabla w)(x) \rrbracket \cdot n & =\sigma(\nabla w)\left(x^{+}\right) \cdot n-\sigma(\nabla w)\left(x^{-}\right) \cdot n \\
& =\sigma(\nabla w)\left(x^{+}\right) \cdot n^{+}+\sigma(\nabla w)\left(x^{-}\right) \cdot n^{-}
\end{aligned}
$$

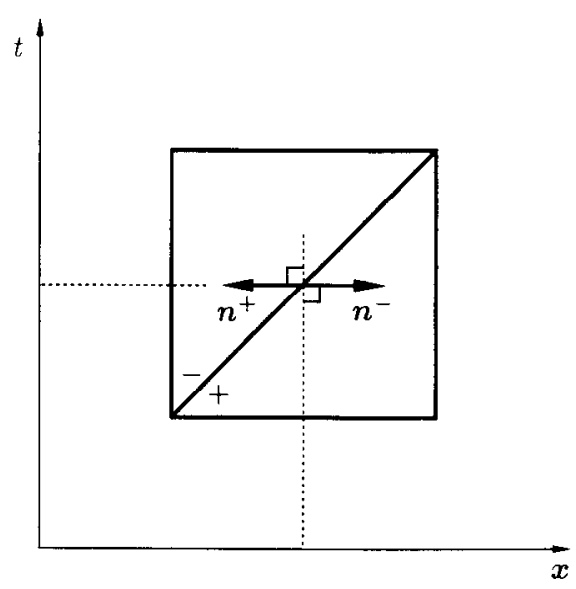

Fig. 8. Illustration of spatial outward normal vectors. 
which demonstrates the invariance of $\llbracket \sigma(\nabla w)(x) \rrbracket \cdot n$ with respect to interchanging the + and designators.

The trial displacement space, $\mathscr{S}^{h}$, and the weighting function space, $\mathscr{V}^{h}$, include $k$ th-order polynomials in both $x$ and $t$. The functions are continuous on each space-time slab, but they may be discontinuous between slabs. Figure 9 depicts a space-time slab containing commonlyused finite elements: linear triangles $(k=1)$ and biquadratic quadrilaterals (9-noded quadrilaterals for which $k=2$ ). The collections of finite element interpolation functions are given by

Trial displacements

$$
\mathscr{S}^{h}=\left\{\boldsymbol{u}^{h}\left|\boldsymbol{u}^{h} \in\left(\mathscr{C}^{0}\left(\bigcup_{n=1}^{N} Q_{n}\right)\right)^{d}, \boldsymbol{u}^{h}\right|_{Q_{n}^{e}} \in\left(\mathscr{P}^{k}\left(Q_{n}^{e}\right)\right)^{d}, \boldsymbol{u}^{h}=\boldsymbol{g} \text { on } Y_{g}\right\}
$$

Displacement weighting functions

$$
\mathscr{V}^{h}=\left\{\boldsymbol{w}^{h}\left|\boldsymbol{w}^{h} \in\left(\mathscr{C}^{0}\left(\bigcup_{n=1}^{N} Q_{n}\right)\right)^{d}, \boldsymbol{w}^{h}\right|_{Q_{n}^{e}} \in\left(\mathscr{P}^{k}\left(Q_{n}^{e}\right)\right)^{d}, \boldsymbol{w}^{h}=0 \text { on } Y_{g}\right\},
$$

where $\mathscr{P}^{k}$ denotes the space of $k$ th-order polynomials and $\mathscr{C}^{0}$ denotes the space of continuous functions.

\subsection{Time-discontinuous Galerkin formulation}

The first component of our space-time finite element method is called the time-discontinuous Galerkin formulation. The objective is to find $\boldsymbol{u}^{h} \in \mathscr{J}^{h}$ such that for all $\boldsymbol{w}^{h} \in \mathscr{V}^{h}$,

where

$$
B_{\mathrm{DG}}\left(\boldsymbol{w}^{h}, \boldsymbol{u}^{h}\right)_{n}=L_{\mathrm{DG}}\left(\boldsymbol{w}^{h}\right)_{n}, \quad n=1,2, \ldots, N,
$$

$$
\begin{aligned}
B_{\mathrm{DG}}\left(\boldsymbol{w}^{h}, \boldsymbol{u}^{h}\right)_{n}= & \left(\dot{\boldsymbol{w}}^{h}, \rho \ddot{\boldsymbol{u}}^{h}\right)_{Q_{n}}+a\left(\dot{\boldsymbol{w}}^{h}, \boldsymbol{u}^{h}\right)_{Q_{n}}+\left(\dot{\boldsymbol{w}}^{h}\left(t_{n-1}^{+}\right), \rho \dot{\boldsymbol{u}}^{h}\left(t_{n-1}^{+}\right)\right)_{\Omega} \\
& +a\left(\boldsymbol{w}^{h}\left(t_{n-1}^{+}\right), \boldsymbol{u}^{h}\left(t_{n-1}^{+}\right)\right)_{\Omega}, \\
L_{\mathrm{DG}}\left(\boldsymbol{w}^{h}\right)_{n}= & \left(\dot{\boldsymbol{w}}^{h}, \boldsymbol{f}\right)_{Q_{n}}+\left(\dot{\boldsymbol{w}}^{h}, \boldsymbol{h}\right)_{\left(Y_{h}\right)_{n}}+\left(\dot{\boldsymbol{w}}^{h}\left(t_{n-1}^{+}\right), \rho \dot{\boldsymbol{u}}^{h}\left(t_{n-1}^{-}\right)\right)_{\Omega} \\
& +a\left(\boldsymbol{w}^{h}\left(t_{n-1}^{+}\right), \boldsymbol{u}^{h}\left(t_{n-1}^{-}\right)\right)_{\Omega} .
\end{aligned}
$$

The three terms evaluated over $Q_{n}$ act to weakly enforce the equation of motion, (7), over the space-time slab; the term evaluated on $\left(Y_{h}\right)_{n}$ acts to weakly enforce the traction boundary condition, (9), while the remaining terms weakly enforce continuity of displacement and velocity between slabs $n$ and $n-1$. This can be seen more clearly from the Euler-Lagrange form of the variational equation

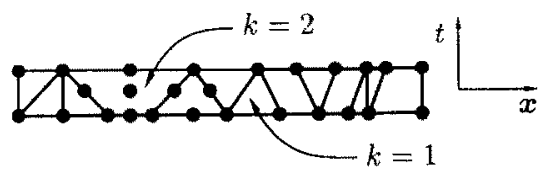

Fig. 9. Illustration of space-time slab discretization; $k$ denotes the order of the interpolation functions. 


$$
\begin{aligned}
& 0=B_{\mathrm{DG}}\left(\boldsymbol{w}^{h}, \boldsymbol{u}^{h}\right)_{n}-L_{\mathrm{DG}}\left(\boldsymbol{w}^{h}\right)_{n} \\
& =\left(\dot{w}^{h}, \mathscr{L} u^{h}-f\right)_{Q_{n}^{s}} \quad \text { (equation of motion) } \\
& +\left(\dot{\boldsymbol{w}}^{h}, \boldsymbol{n} \cdot \llbracket \boldsymbol{\sigma}\left(\nabla \boldsymbol{u}^{h}\right)(\boldsymbol{x}) \rrbracket\right)_{Y_{n}^{\Sigma}} \quad \text { (traction continuity in space) } \\
& +\left(\dot{\boldsymbol{w}}^{h}, \boldsymbol{n} \cdot \boldsymbol{\sigma}\left(\nabla \boldsymbol{u}^{h}\right)-\boldsymbol{h}\right)_{\left(\Upsilon_{\boldsymbol{h}}\right)_{n}} \text { (traction boundary condition) } \\
& +\left(\dot{w}^{h}\left(t_{n-1}^{+}\right), \rho \llbracket \dot{u}^{h}\left(t_{n-1}\right) \rrbracket\right)_{\Omega} \text { (velocity continuity in time) } \\
& +a\left(w^{h}\left(t_{n-1}^{+}\right), \llbracket u^{h}\left(t_{n-1}\right) \rrbracket\right)_{\Omega} \text { (displacement continuity in time), }
\end{aligned}
$$

where we have used the integration-by-parts formula

$$
\begin{aligned}
a\left(\dot{\boldsymbol{w}}^{h}, \boldsymbol{u}^{h}\right)_{Q_{n}}= & -\left(\dot{\boldsymbol{w}}^{h}, \nabla \cdot \boldsymbol{\sigma}\left(\nabla \boldsymbol{u}^{h}\right)\right)_{Q_{n}^{\Sigma}}+\left(\dot{\boldsymbol{w}}^{h}, \boldsymbol{n} \cdot \llbracket \boldsymbol{\sigma}\left(\nabla \boldsymbol{u}^{h}\right)(\boldsymbol{x}) \rrbracket\right)_{Y_{n}^{\Sigma}} \\
& +\left(\dot{\boldsymbol{w}}^{h}, \boldsymbol{n} \cdot \boldsymbol{\sigma}\left(\nabla \boldsymbol{u}^{h}\right)\right)_{\left(Y_{h}\right)_{n}}
\end{aligned}
$$

There are several important consequences of using time-discontinuous functions and the temporal jump operators:

(1) The equations to be solved for each slab are decoupled from those of the other slabs. The data from the end of the previous time slab are employed as initial conditions for the current slab.

(2) The jump operators introduce numerical dissipation into the formulation. They may be considered as sophisticated 'artificial viscosities' but they do not inherit the deficiencies of the classical artificial viscosities. Since they are form-invariant with respect to the order of element interpolations, the resultant algorithms are higher-order accurate; see [76].

(3) Displacement continuity is weakly enforced via the strain-energy inner product, $a(\cdot, \cdot)_{\Omega}$. This is the crucial element enabling generalization of the time-discontinuous space-time finite element methods successfully developed for first-order systems to second-order hyperbolic equations.

From (39), it follows that a sufficiently smooth exact solution of the initial/boundary-value problem, $\boldsymbol{u}$, satisfies

$$
B_{\mathrm{DG}}\left(\boldsymbol{w}^{h}, \boldsymbol{u}\right)_{n}=L_{\mathrm{DG}}\left(\boldsymbol{w}^{h}\right)_{n}
$$

for all $w^{h} \in \mathscr{V}^{h}$ and $n=1,2, \ldots, N$. In finite element terminology, this is an appropriate notion of consistency for the time-discontinuous Galerkin formulation; it implies that higherorder accurate algorithms can be developed by choosing higher-order finite element interpolations.

Time-discontinuous Galerkin formulations lead to systems of equations of the form

$$
\boldsymbol{K} \boldsymbol{d}=\boldsymbol{F}
$$

where $\boldsymbol{d}$ is the vector of unknown nodal displacements of a slab. For cach space-time slab, one such system of equations needs to be solved; the algorithm proceeds by solving each successive 


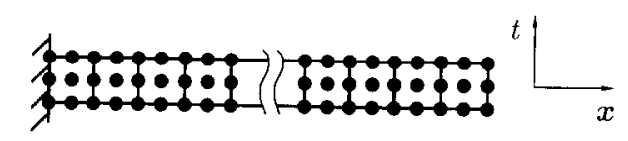

Fig. 10. Space-time finite element discretization for the bar impact problem using 200 9-noded quadrilateral elements.

system. Thus, the space-time finite element formulation obviates the need for a separate time-integration algorithm as required in the semidiscrete approach. One disadvantage of the time-discontinuous space-time finite element method is that it leads to a larger system of equations than those produced by typical semidiscrete methods. We have developed predictormulticorrector algorithms, based on the time-discontinuous Galerkin method, to reduce the computational costs associated with solving the larger system of equations, see [91] for details.

To demonstrate the improved performance of the time-discontinuous Galerkin method when compared with standard semidiscrete algorithms, the one-dimensional bar impact problem was solved using 2009 -noded quadrilateral space-time elements per slab, as shown in Fig. 10. The 9-noded quadrilateral element permits quadratic variation of displacement in both space and time. This mesh was used for all the space-time finite element formulations. The stress distribution in the bar as shown in Fig. 11 was computed using the timediscontinuous Galerkin method. There are a few oscillations in front of the discontinuity and a small oscillation behind the discontinuity, but the computed response is substantially better than those computed using the trapezoidal and HHT- $\alpha$ semidiscrete methods. Note also that the discontinuity is captured quite well; that is, the slope of the computed solution is fairly steep and is not overly smeared. (The support of the computed discontinuity is 9 nodes.)

\subsection{Galerkin/least-squares formulation}

It is desirable to further reduce or eliminate the oscillations in the computed response. In addition, it is useful to prove that the space-time finite element formulation converges for

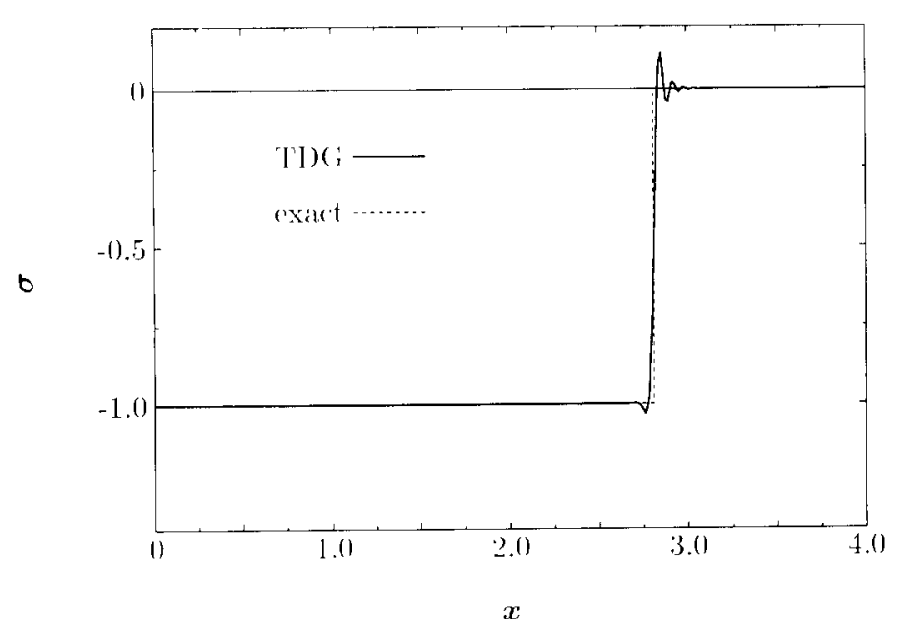

Fig. 11. Bar impact problem. Stress distribution calculated using time-discontinuous Galerkin algorithm. 
arbitrary space-time discretizations and higher-order element interpolations. To achieve these goals, additional stabilizing operators are added to the time-discontinuous Galerkin method. These stabilizing operators have least-squares form; the resultant formulation is called the Galerkin/least-squares space-time finite element method and is given by

where

$$
B_{\mathrm{GLS}}\left(\boldsymbol{w}^{h}, \boldsymbol{u}^{h}\right)_{n}=L_{\mathrm{GLS}}\left(\boldsymbol{w}^{h}\right)_{n}, \quad n=1,2, \ldots, N,
$$

$$
\begin{aligned}
& B_{\mathrm{GLS}}\left(\boldsymbol{w}^{h}, \boldsymbol{u}^{h}\right)=B_{\mathrm{DG}}\left(\boldsymbol{w}^{h}, \boldsymbol{u}^{h}\right)_{n}+\left(\mathscr{L} \boldsymbol{w}^{h}, \rho^{-1} \boldsymbol{\tau} \mathscr{L} \boldsymbol{u}^{h}\right)_{Q \frac{\Sigma}{n}} \\
& +\left(\boldsymbol{n} \cdot \llbracket \boldsymbol{\sigma}\left(\nabla \boldsymbol{w}^{h}\right)(\boldsymbol{x}) \rrbracket, \rho^{-1} \boldsymbol{s} \boldsymbol{n} \cdot \llbracket \boldsymbol{\sigma}\left(\nabla \boldsymbol{u}^{h}\right)(\boldsymbol{x}) \rrbracket\right)_{Y_{n}^{\Sigma}}+\left(\boldsymbol{n} \cdot \boldsymbol{\sigma}\left(\nabla \boldsymbol{w}^{h}\right), \rho^{-1} \boldsymbol{s n} \cdot \boldsymbol{\sigma}\left(\nabla \boldsymbol{u}^{h}\right)\right)_{\left(Y_{h}\right)_{n}}, \\
& L_{\mathrm{GLS}}\left(\boldsymbol{w}^{h}\right)_{n}=L_{\mathrm{DG}}\left(\boldsymbol{w}^{h}\right)_{n}+\left(\mathscr{L} \boldsymbol{w}^{h}, \rho^{-1} \tau f\right)_{Q_{n}^{\Sigma}}+\left(\boldsymbol{n} \cdot \boldsymbol{\sigma}\left(\nabla \boldsymbol{w}^{h}\right), \rho^{-1} \boldsymbol{s h}\right)_{\left(Y_{h}\right)_{n}} .
\end{aligned}
$$

The matrices $\tau$ and $s$ have dimensions of time and slowness, respectively; both are $d \times d$ matrices. The corresponding least-squares terms add stability without degrading the accuracy of the underlying time-discontinuous Galerkin method.

The stress distribution computed using the Galerkin/least-squares method is shown in Fig. 12 for $t=2.81$. Compared to the solution obtained using the time-discontinuous Galerkin method, the number of oscillations in front of the discontinuity has been reduced; there is still a small overshoot in front of and a small undershoot behind the discontinuity. The slope of the discontinuity is accurately captured; the discontinuity support is 14 nodes.

\subsection{Discontinuity-capturing formulation}

To completely eliminate oscillations in the computed solution requires using a nonlinear algorithm even for this linear problem. It is well-known that a monotone (non-oscillatory) response for discontinuous solutions cannot be computed using a high-order accurate (accuracy greater than first-order) linear algorithm. Since the Galerkin/least-squares method with

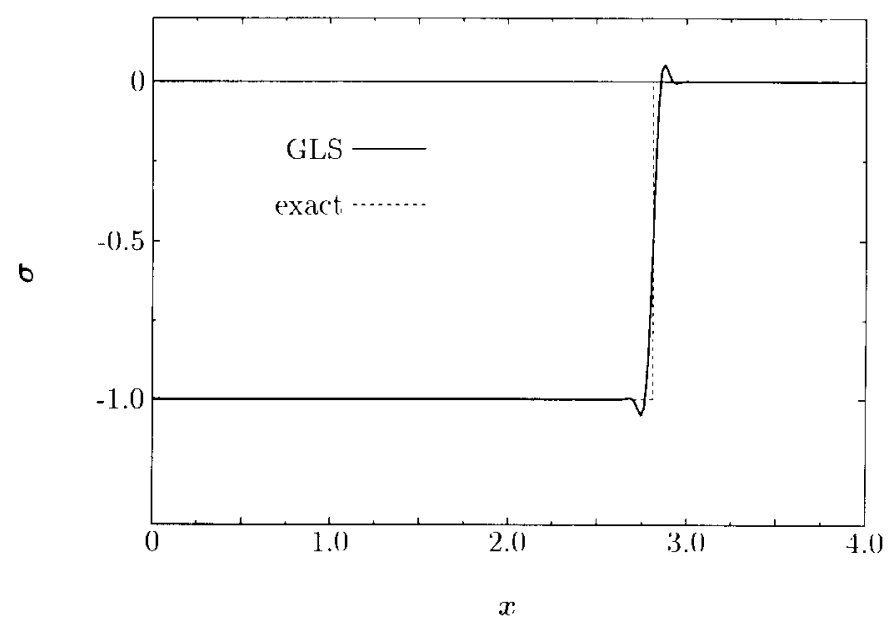

Fig. 12. Bar impact problem. Stress distribution calculated using Galerkin/least-squares algorithm. 
9-noded quadrilateral elements is a higher-order accurate scheme, nonlinear discontinuitycapturing operators were designed to provide better stability in regions of sharp gradients. The discontinuity-capturing formulation is given by

where

$$
B\left(\boldsymbol{w}^{h}, \boldsymbol{u}^{h}\right)_{n}=L\left(\boldsymbol{w}^{h}\right)_{n}, \quad n=1,2, \ldots, N,
$$

and

$$
\begin{aligned}
& B\left(\boldsymbol{w}^{h}, \boldsymbol{u}^{h}\right)_{n}=B_{\mathrm{GLS}}\left(\boldsymbol{w}^{h}, \boldsymbol{u}^{h}\right)_{n}+\int_{Q_{n}^{\Sigma}} \frac{\left(\boldsymbol{R}^{h} \cdot \rho^{-1} \boldsymbol{\tau} \boldsymbol{R}^{h}\right)}{\left|\boldsymbol{\nabla}_{\boldsymbol{\xi}^{2}} \boldsymbol{u}^{h}\right|^{2}} \nabla_{\boldsymbol{\xi}^{2}} \boldsymbol{w}^{h} \cdot \nabla_{\boldsymbol{\xi}^{2}} \boldsymbol{u}^{h} \mathrm{~d} Q, \\
& L\left(\boldsymbol{w}^{h}\right)_{n}=L_{\mathrm{GLS}}\left(\boldsymbol{w}^{h}\right)_{n}
\end{aligned}
$$

$$
\begin{aligned}
& \boldsymbol{R}^{h}=\mathscr{L} \boldsymbol{u}^{h}-\boldsymbol{f}, \\
& \boldsymbol{\nabla}_{\xi^{2}} \boldsymbol{w}^{h}=\left\{\left\{\frac{\partial^{2} \boldsymbol{w}^{h}}{\partial \xi_{0}^{2}}\right\}^{\mathrm{t}}\left\{\frac{\partial^{2} \boldsymbol{w}^{h}}{\partial \xi_{1}^{2}}\right\}^{\mathrm{t}}\left\{\frac{\partial^{2} \boldsymbol{w}^{h}}{\partial \xi_{2}^{2}}\right\}^{\mathrm{t}} \cdots\left\{\frac{\partial^{2} \boldsymbol{w}^{h}}{\partial \xi_{d}^{2}}\right\}^{\mathrm{t}}\right\}^{\mathrm{t}} .
\end{aligned}
$$

The discontinuity-capturing operator has several important features. For each element, it is proportional to the square of the residual, $\left(\boldsymbol{R}^{h} \cdot \rho^{-1} \tau \boldsymbol{R}^{h}\right)$. If the solution is smooth, the solution gradients and residual are small. Thus, the effect of the discontinuity-capturing term also is small and the formulation is essentially equivalent to the Galerkin/least-squares method. In regions where the solution gradients are large, the residual also is large and the effect of the additional operator becomes important. In these regions, the discontinuitycapturing operator adds stability by controlling the local second derivatives of the solution, $\left(\nabla_{\xi^{2}} \boldsymbol{w}^{h} \cdot \nabla_{\xi^{2}} \boldsymbol{u}^{h}\right)$; note that both spatial and temporal second derivatives are controlled. Since the discontinuity-capturing operator is dependent on the element residual, the resultant formulation is nonlinear even for the problem of linear elastodynamics. When solving nonlinear elastodynamics problems, the nonlinear discontinuity-capturing algorithm does not increase computational costs, when compared to the Galerkin/least-squares method, since the underlying equations are inherently nonlinear.

Figure 13 shows the stress distribution in the elastic bar for $t=2.81$ computed using the

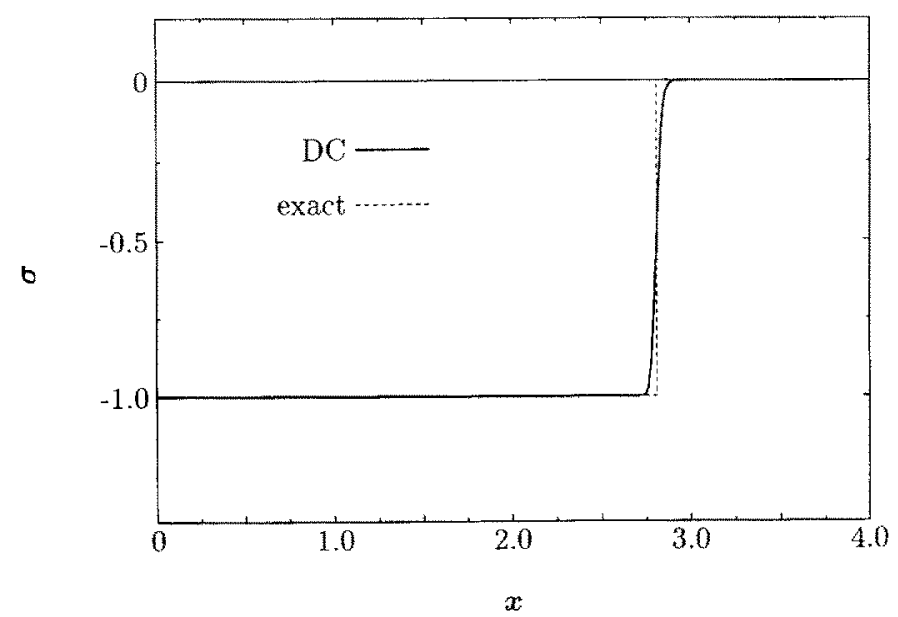

Fig. 13. Bar impact problem. Stress distribution calculated using discontinuity-capturing algorithm. 


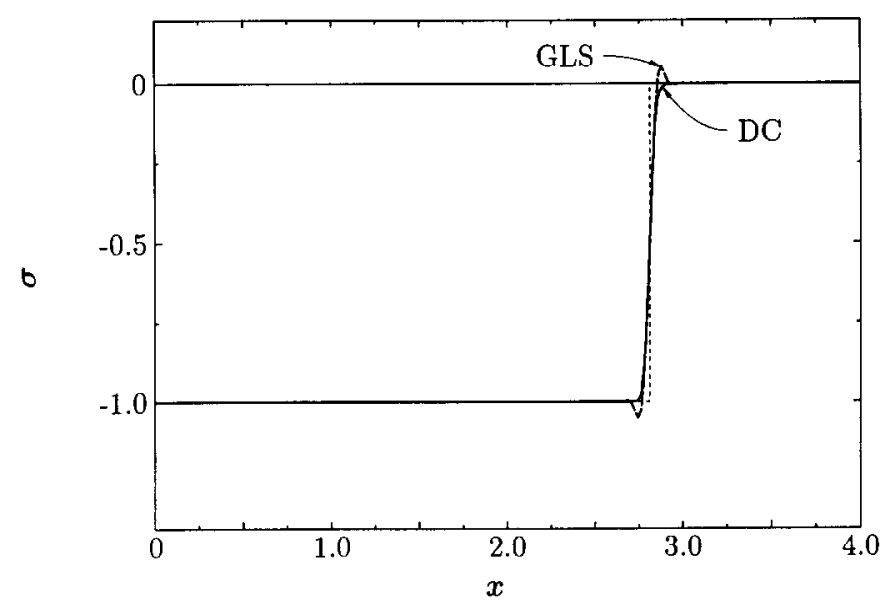

Fig. 14. Bar impact problem. Comparison of stress distribution calculated using Galerkin/least-squares (GLS) and discontinuity-capturing (DC) algorithms.

discontinuity-capturing formulation. Note that the oscillations near the discontinuity have been eliminated. It is useful to compare the solutions obtained by the Galerkin/least-squares and discontinuity-capturing formulations; see Fig. 14. We again note that the discontinuitycapturing formulation has eliminated the few remaining oscillations. It may also be observed that the discontinuity-capturing operator does not 'smear' the discontinuity; that is, near the discontinuity, the solutions obtained by both methods are virtually identical. This result emphasizes that the proposed discontinuity-capturing operator acts like a higher-order accurate artificial viscosity.

\section{Stability and convergence analyses}

In this section, we shall present results from stability and convergence analyses of the space-time finite element formulations presented in the previous section. See [91] for details of the analyses.

For linear elastodynamics, a natural measure of stability is the total energy, given by

$$
\mathscr{E}\left(\boldsymbol{w}^{h}\right)=\frac{1}{2}\left(\dot{\boldsymbol{w}}^{h}, \rho \dot{w}^{h}\right)_{\Omega}+\frac{1}{2} a\left(\boldsymbol{w}^{h}, \boldsymbol{w}^{h}\right)_{\Omega}
$$

In the absence of forcing terms, i.e., $f=g=\boldsymbol{h}=\mathbf{0}$ in (7)-(9), we have proved that the following energy decay inequality holds for the three space-time finite element formulations presented in this paper:

$$
\mathscr{E}\left(\boldsymbol{u}^{h}\left(T^{-}\right)\right) \leqslant \mathscr{E}(\boldsymbol{u}(0)),
$$

where $\mathscr{E}(u(0))$ is the initial total energy. In other words, the space-time finite element formulations are unconditionally stable for linear elastodynamics.

To study the convergence rates of the space-time finite element formulations, an appropriate space-time mesh parameter is given by 


$$
h=\max \{c \Delta t, \Delta x\},
$$

where $c$ is the dilatational wave speed and $\Delta x$ and $\Delta t$ are maximum element diameters in space and time, respectively. To study the convergence properties of the formulations, the spatial and temporal domains simultaneously are refined. (The mesh parameter, $h$, is decreasing uniformly in $\Delta x$ and $\Delta t$.) Assuming the exact solution to (7)-(11) is sufficiently smooth in the sense that

$$
u \in\left(H^{k+1}(Q)\right)^{d},
$$

then, for the Galerkin/least-squares formulation, (43),

$$
\|\boldsymbol{e} \mid\|^{2} \leqslant C(\boldsymbol{u}) h^{2 k-1},
$$

where $k$ is the order of the finite element interpolation,

$$
\boldsymbol{e}=\boldsymbol{u}^{h}-\boldsymbol{u}
$$

is the error, $C(\boldsymbol{u})$ is independent of $h$ and

$$
\begin{aligned}
\|\boldsymbol{e}\| \|^{2}= & \mathscr{E}\left(\boldsymbol{e}\left(T^{-}\right)\right)+\mathscr{E}\left(\boldsymbol{e}\left(0^{+}\right)\right)+\sum_{n=1}^{N-1} \mathscr{E}\left(\llbracket \boldsymbol{e}\left(t_{n}\right) \rrbracket\right) \\
+\sum_{n=1}^{N} & \left\{\left(\mathscr{L} \boldsymbol{e}, \rho^{-1} \boldsymbol{\tau} \mathscr{L} \boldsymbol{e}\right)_{Q_{n}^{\Sigma}}+\left(\boldsymbol{n} \cdot \llbracket \boldsymbol{\sigma}(\nabla \boldsymbol{e})(\boldsymbol{x}) \rrbracket, \rho^{-1} \boldsymbol{s} \boldsymbol{n} \cdot \llbracket \boldsymbol{\sigma}(\nabla \boldsymbol{e})(\boldsymbol{x}) \rrbracket\right)_{Y_{n}^{\Sigma}}\right. \\
& \left.+\left(\boldsymbol{n} \cdot \boldsymbol{\sigma}(\nabla \boldsymbol{e}), \rho^{-1} \boldsymbol{s} \boldsymbol{n} \cdot \boldsymbol{\sigma}(\nabla \boldsymbol{e})\right)_{\left(Y_{h}\right)_{h}}\right\}
\end{aligned}
$$

is a norm that is stronger than the total energy norm, (51).

As measured in the \|\|$\cdot \|||$-norm, the convergence rate proved for the Galerkin/least-squares method is sharp (in the sense that the interpolation error has the same rate of convergence, $h^{2 k-1}$, when measured in the $\||\cdot|||$-norm; see [92] for details of interpolation error theory for finite elements).

For the discontinuity-capturing formulation, (46), optimal convergence, (55), has been proved assuming the solution is sufficiently smooth in the sense that

$$
\max \left(\boldsymbol{R}^{h} \cdot \rho^{-1} \boldsymbol{\tau} \boldsymbol{R}^{h}\right) \leqslant C h^{2},
$$

where the maximum is taken over all space-time finite elements and $C$ is independent of $h$.

To numerically evaluate convergence rates, the response of a one-dimensional, homogeneous elastic rod was calculated. Both ends of the rod were fixed; no external loads were applied; the initial velocity was zero; the initial displacement was proportional to the first harmonic. Unit values were specified for the length, area, density and elastic coefficient of the rod. The response was calculated for the time interval $0 \leqslant t \leqslant T=1.2$. Figure 15 shows the error computed using the Galerkin/least-squares and discontinuity-capturing formulations 


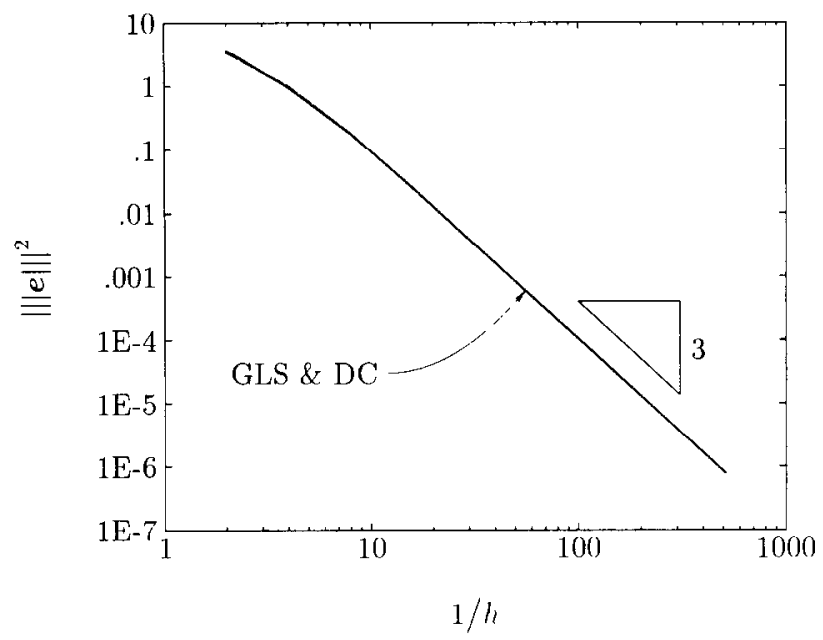

Fig. 15. Calculation of numerical error using Galerkin/least-squares (GLS) and discontinuity-capturing (DC) formulations; $h$ is the spatial distance between adjacent nodes.

employing biquadratic elements. Both formulations achieve the cubic rate of convergence predicted by (55). These results also emphasize that the discontinuity-capturing operators presented in Section 3.4 do not degrade the underlying accuracy of the time-discontinuous Galerkin formulation for problems with smooth solutions.

\section{Conclusions}

We have developed new space-time finite element formulations for elastodynamics which generate unconditionally stable, higher-order accurate algorithms. Included in the formulations is a nonlinear discontinuity-capturing operator which controls oscillations induced by sharp gradients or discontinuities in the solution without degrading the accuracy of the Galerkin/least-squares method in smooth regions of the solution. The additional stabilizing terms in the space-time formulations were derived from mathematical analyses; consequently, the resultant space-time finite element methods possess a firm mathematical foundation.

Figure 16 summarizes the numerical results presented in this paper. Starting with the trapezoidal rule algorithm, which is known to have difficulties limiting oscillations due to high frequency response, researchers added numerical dissipation to control unwanted oscillations when solving structural dynamics problems, e.g., the HHT- $\alpha$ is an effective structural dynamics algorithm that provides numerical damping. Even with the added numerical damping, there still exists a number of oscillations in the solution; these results are indicative of the performance of structural dynamics algorithms when used to solve wave propagation problems. Using the time-discontinuous Galerkin method, the oscillations are greatly reduced. Adding least-squares operators to the formulation further reduces the number of oscillations. To eliminate the oscillations required adding a nonlinear discontinuity-capturing operator. The discontinuity-capturing formulation results in a monotone solution for the bar impact problem with excellent resolution of the stress discontinuity. 


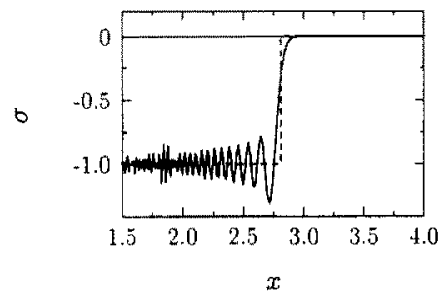

Trapezoidal

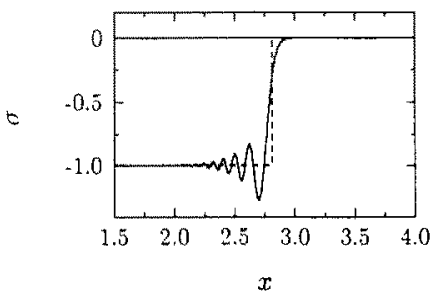

Hilber-Hughes-Taylor

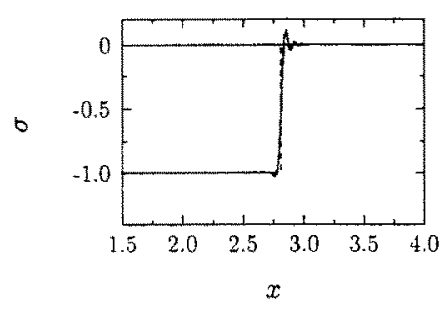

Time-discontinuous Galerkin

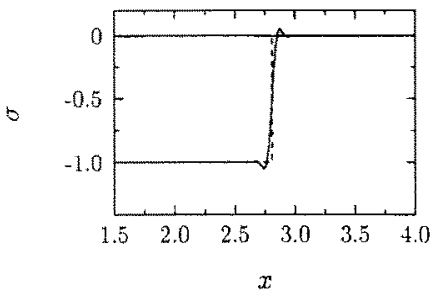

Galerkin/least-squares

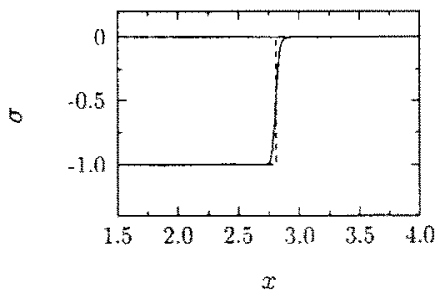

Discontinuity-capturing

Fig. 16. Bar impact problem. Comparison of results.

While the numerical results presented in this paper have been limited to one-dimensional problems, the formulations are applicable to multi-dimensional problems; research efforts are underway to address the implementational issues of the multi-dimensional formulation. One area of great potential for space-time finite element methods is the development of adaptive mesh refinement strategies in both space and time. Johnson and colleagues have recently developed very interesting adaptive mesh refinement strategies for first-order hyperbolic and parabolic equations based on the space-time finite element method. Having laid the foundation for space-time finite element methods for second-order hyperbolic equations, we feel there is considerable potential for future applications in elastodynamics, structural dynamics and second-order hyperbolic systems in general.

\section{References}

[1] H.M. Hilber, Analysis and design of numerical integration methods in structural dynamics, Ph.D. Thesis, Earthquake Engineering Research Center, University of California, Berkeley, 1976.

[2] H.M. Hilber, T.J.R. Hughes and R.L. Taylor, Improved numerical dissipation for time integration algorithms in structural dynamics, Earthquake Engrg. Struct. Dyn. 5 (1977) 283-292. 
[3] H.M. Hilber and T.J.R. Hughes, Collocation, dissipation and 'overshoot' for time integration schemes in structural dynamics, Earthquake Engrg. Struct. Dyn. 6 (1978) 99-118.

[4] T.J.R. Hughes, Analysis of transient algorithms with particular reference to stability behavior, in: T. Belytschko and T.J.R. Hughes, eds., Computational Methods for Transient Ananalysis (North-Holland, Amsterdam, 1983) 67-155.

[5] T.J.R. Hughes, The Finite Element Method: Linear Static and Dynamic Finite Element Analysis (PrenticeHall, Englewood Cliffs, NJ, 1987).

[6] P. Hansbo, Adaptivity and streamline diffusion procedures in the finite element method, Ph.D. Thesis, Department of Structural Mechanics, Chalmers University of Technology, Göteborg, Sweden, 1989.

[7] J.H. Argyris and D.W. Scharpf, Finite elements in time and space, Nucl. Engrg. Des. 10 (1969) 456-464.

[8] I. Fried, Finite element analysis of time-dependent phenomena, AIAA J. 7 (1969) 1170-1173.

[9] J.T. Oden, A general theory of finite elements II. Applications, Internat. J. Numer. Methods in Engrg. 1 (1969) 247-259.

[10] M.E. Gurtin, Variational principles for linear initial value problems, Quart. Appl. Math. 22 (1964) 252-256.

[11] C.D. Bailey, Application of Hamilton's law of varying action, AIAA J. 13 (1975) 1154-1157.

[12] C.D. Bailey, The method of Ritz applied to the equations of Hamilton, Comput. Methods Appl. Mech. Engrg. 7 (1976) 235-247.

[13] M. Baruch and R. Riff, Hamilton's principle, Hamilton's law $-6^{n}$ correct formulations, AlAA J. 20 (1982) $687-692$.

[14] M. Borri, G.L. Ghiringhelli, M. Lanz, P. Mantegazza and T. Merlini, Dynamic response of mechanical systems by a weak Hamiltonian formulation, Comput. \& Structures 20 (1985) 495-508.

[15] G.F. Howard and J.E.T. Penny, The accuracy and stability of time domain finite element solutions, J. Sound Vibration 61 (1978) 585-595.

[16] R.E. Nickell and J.L. Sackman, Approximate solutions in linear, coupled thermoelasticity, J. Appl. Mech. 35 (1968) 255-266.

[17] R. Riff and M. Baruch, Stability of time finite elements, AIAA J. 22 (1984) 1171-1173.

[18] R. Riff and M. Baruch, Time finite element discretization of Hamilton's law of varying action, AIAA J. 22 (1984) $1310-1318$.

[19] T.E. Simkins, Unconstrained variational statements for initial and boundary-value problems, AIAA J. 16 (1978) 559-563.

[20] T.E. Simkins, Finite elements for initial value problems in dynamics, AIAA J. 19 (1981) 1357-1362.

[21] D.R. Smith and C.V. Smith, Jr., When is Hamilton's principle an extremum principle?, AIAA J. 12 (1974) $1573-1576$.

[22] C.V. Smith, Jr., Discussion on 'Hamilton, Ritz and plastodynamics', J. Appl. Mech. 44 (1977) 796-797.

[23] E.L. Wilson and R.E. Nickell, Application of finite element method to heat conduction analysis, Nucl. Engrg. Des. 4 (1966) 276-286.

[24] J.R. Yu and T.R. Hsu, Analysis of heat conduction in solids by space-time finite element method, Internat. J. Numer. Methods Engrg. 21 (1985) 2001-2012.

[25] D.A. Peters and A.P. Izadpanah, $h p$-version finite elements for the space-time domain, Comput. Mech. 3 (1988) 73-88.

[26] C. Bajer, Triangular and tetrahedral space-time finite elements in vibrational analysis, Internat. J. Numer. Methods Engrg. 23 (1986) 2031-2048.

[27] C. Bajer, Notes on the stability of non-rectangular space-time finite elements, Internat. J. Numer. Methods Engrg. 24 (1987) 1721-1739.

[28] R. Bonnerot and P. Jamet, A second order finite element method for the one-dimensional Stefan problem, Internat. J. Numer. Methods Engrg. 8 (1974) 811-820.

[29] R. Bonnerot and P. Jamet, Numerical computation of the free boundary for the two-dimensional Stefan problem by space-time finite elements, J. Comput. Phys. 25 (1977) 163-181.

[30] J.C. Bruch and G. Zyvoloski, A finite element weighted residual solution to one-dimensional field problems, Internat. J. Numer. Methods Engrg. 6 (1973) 577-585.

[31] J.C. Bruch and G. Zyvoloski, Transient two-dimensional heat conduction problems solved by the finite element method, Internat. J. Numer. Methods Engrg. 8 (1974) 481-494.

[32] M. Morandi Cecchi and A. Cella, A Ritz-Galerkin approach to heat conduction: method and results, in: Proceedings of the Fourth Canadian Congress of Applied Mechanics, Session H (Montreal, 1973) 767-768. 
[33] A. Cella and M. Lucchesi, Space-time finite elements for the wave propagation problem, Meccanica 10 (1975) $168-170$.

[34] A. Cella, M. Lucchesi and G. Pasquinelli, Space-time elements for the shock wave propagation problem, Internat. J. Numer. Methods Engrg. 15 (1980) 1475-1488.

[35] Y.K. Cheung and L.G. Tham, Time-space finite elements for unsaturated flow through porous media, in: Proceedings of the Third International Conference on Numerical Methods in Geomechanics, Vol. 1 (A.A. Balkema, Rotterdam, 1979) 251-256.

[36] K.S. Chung, The fourth-dimension concept in the finite element analysis of transient heat transfer problems, Internat. J. Numer. Methods Engrg. 17 (1981) 315-325.

[37] P. Jamet and R. Bonnerot, Numerical solution of the Eulerian equations of compressible flow by a finite element method which follows the free boundary and the interfaces, J. Comput. Phys. 18 (1975) 21-45.

[38] Z. Kacprzyk and T. Lewiński, Comparison of some numerical integration methods for the equations of motion of systems with a finite number of degrees of freedom, Engrg. Transactions 31 (1983) 213-240.

[39] A.W.M. Kok, Pulses in finite elements, in: Proceedings of the International Conference on Computing in Civil Engineering (ASCE, New York, 1981) 286-301.

[40] D.L. Lewis, J. Lund and K.L. Bowers, The space-time Sinc-Galerkin method for parabolic problems, Internat. J. Numer. Methods Engrg. 24 (1987) 1629-1644.

[41] H. Nguyen and J. Reynen, A space-time least-square finite element scheme for advection-diffusion equations, Comput. Methods Appl. Mech. Engrg. 42 (1984) 331-342.

[42] A.C. Papanastasiou, L.E. Scriven and C.W. Macosko, Bubble growth and collapse in viscoelastic liquids analyzed, J. Non-Newtonian Fluid Mech. 16 (1984) 53-75.

[43] E. Varoglu and W.D.L. Finn, Space-time finite elements incorporating characteristics for the Burgers' equation, Internat. J. Numer. Methods Engrg. 16 (1980) 171-184.

[44] J.H. Argyris, L.E. Vaz and K.J. Willam, Higher order methods for transient diffusion analysis, Comput. Methods Appl. Mech. Engrg. 12 (1977) 243-278.

[45] M. Kawahara and K. Hasegawa, Periodic Galerkin finite element method of tidal flow, Internat. J. Numer. Methods Engrg. 12 (1978) 115-127.

[46] J. Kujawski and C.S. Desai, Generalized time finite element algorithm for non-linear dynamic problems, Engrg. Computations 1 (1984) 247-251.

[47] O.C. Zienkiewicz and C.J. Parekh, Transient field problems-Two and three dimensional analysis by isoparametric finite elements, Internat. J. Numer. Methods Engrg. 2 (1970) 61-71.

[48] O.C. Zienkiewicz, The Finite Element Method (McGraw-Hill, London, 1977).

[49] O.C. Zienkiewicz, A new look at the Newmark, Houbolt and other time stepping formulas. A weighted residual approach, Earthquake Engrg. Struct. Dyn. 5 (1977) 413-418.

[50] W.H. Reed and T.R. Hill, Triangular mesh methods for the neutron transport equation, Report LA-UR-73479, Los Alamos Scientific Laboratory, Los Alamos, 1973.

[51] P. Lesaint and P.-A. Raviart, On a finite element method for solving the neutron transport equation, in: C. de Boor ed., Mathematical Aspects of Finite Elements in Partial Differential Equations (Academic Press, New York, 1974) 89-123.

[52] R. Bonnerot and P. Jamet, A third order accurate discontinuous finite element method for the onedimensional Stefan problem, J. Comput. Phys. 32 (1979) 145-167.

[53] M. Delfour, W. Hager and F. Trochu, Discontinuous Galerkin methods for ordinary differential equations, Math. Comp. 36 (1981) 455-473.

[54] T.J.R. Hughes, L.P. Franca and M. Mallet, A new finite element formulation for computational fluid dynamics: VI. Convergence analysis of the generalized SUPG formulation for linear time-dependent multidimensional advective-diffusive systems, Comput. Methods Appl. Mech. Engrg. 63 (1987) 97-112.

[55] T.J.R. Hughes, L.P. Franca, G.M. Hulbert, Z. Johan and F. Shakib, The Galerkin/least-squares method for advective-diffusive equations, in: T.J.R. Hughes and T.E. Tezduyar, eds., Recent Developments in Computational Fluid Dynamics, ADM Vol. 95 (ASME, New York, 1988) 75-99.

[56] P. Jamet, Galerkin-type approximations which are discontinuous in time for parabolic equations in a variable domain, SIAM J. Numer. Anal. 15 (1978) 912-928.

[57] C. Johnson, U. Nävert and J. Pitkäranta, Finite element methods for linear hyperbolic problems, Comput. Methods Appl. Mech. Engrg. 45 (1984) 285-312.

[58] C. Johnson and J. Pitkäranta, An analysis of the discontinuous Galerkin method for a scalar hyperbolic 
equation, Technical Report MAT-A215, Institute of Mathematics, Helsinki University of Technology, Helsinki, Finland, 1984.

[59] C. Johnson, Streamline diffusion methods for problems in fluid mechanics, in: R.H. Gallagher, G.F. Carey, J.T. Oden and O.C. Zienkiewicz, eds., Finite Elements in Fluids-Vol. 6 (Wiley, Chichester, 1986) 251-261.

[60] C. Johnson and J. Saranen, Streamline diffusion methods for the incompressible Euler and Navier-Stokes equations, Math. Comp. 47 (1986) 1-18.

[61] C. Johnson, Numerical Solutions of Partial Differential Equations by the Finite Element Method (Cambridge Univ. Press, Cambridge, 1987).

[62] U. Nävert, A finite element method for convection-diffusion problems, Ph.D. Thesis, Department. of Computer Science, Chalmers University of Technology, Göteborg, Sweden, 1982.

[63] F. Shakib, Finite element analysis of the compressible Euler and Navier-Stokes equations, Ph.D. Thesis, Division of Applied Mechanics, Stanford University, Stanford, California, 1989.

[64] V. Thomée, Galerkin Finite Element Methods for Parabolic. Problems (Springer, New York, 1984).

[65] C. Johnson, Error estimates and automatic time step control for numerical methods for stiff ordinary differential equations, Technical Report 1984-27, Department of Mathematics, Chalmers University of Technology and the University of Göteborg, Göteborg, Sweden, 1984.

[66] K. Eriksson, C. Johnson and J. Lennblad, Optimal error estimates and adaptive time and space step control for linear parabolic problems, Technical Report 1986-06, Department of Mathematics, Chalmers University of Technology and the University of Göteborg, Göteborg, Sweden, 1986.

[67] K. Eriksson and C. Johnson, Error estimates and automatic time step control for nonlinear parabolic problems, I, SIAM J. Numer. Anal. 24 (1987) 12-23.

[68] K. Eriksson and C. Johnson, Adaptive finite element methods for parabolic problems: I. A linear model problem, Technical Report 1988-31, Department of Mathematics, Chalmers University of Technology and the University of Göteborg, Göteborg, Sweden, 1988.

[69] C. Johnson, Y.-Y. Nie and V. Thomée, An a posteriori error estimate and automatic time step control for a backward Euler discretization of a parabolic problem, Technical Report 1985-23, Department of Mathematics, Chalmers University of Technology and the University of Göteborg, Göteborg, Sweden, 1985.

[70] C. Johnson and A. Szepessy, On the convergence of streamline diffusion finite element methods for hyperbolic conservation laws, in: T.E. Tezduyar and T.J.R. Hughes, eds., Numerical Methods for Compressible Flows-Finite Difference, Element and Volume Techniques, AMD Vol. 78 (ASME, New York, 1986) 75-91.

[71] C. Johnson and A. Szepessy, On the convergence of a finite element method for a nonlinear hyperbolic conservation law, Math. Comp. 49 (1987) 427-444.

[72] C. Johnson, A. Szepessy and P. Hansbo, On the convergence of shock-capturing streamline diffusion finite element methods for hyperbolic conservation laws, Technical Report 1987-21, Department of Mathematics, Chalmers University of Technology and the University of Göteborg, Göteborg, Sweden, 1987.

[73] A. Szepessy, Convergence of the streamline diffusion finite element method for conservation laws, Ph.D. Thesis, Department of Mathematics, Chalmers University of Technology, Göteborg, Sweden, 1989.

[74] T.J.R. Hughes and J.E. Marsden, Classical elastodynamics as a linear symmetric hyperbolic system, J. Elasticity 8 (1978) 97-110.

[75] F. John, Finite amplitude waves in a homogeneous isotropic elastic solid, Commun. Pure Appl. Math. 30 (1977) 421-446.

[76] T.J.R. Hughes and G.M. Hulbert, Space-time finite element methods for elastodynamics: Formulations and error estimates, Comput. Methods Appl. Mech. Engrg. 66 (1988) 339-363.

[77] T.J.R. Hughes and M. Mallet, A new finite element formulation for computational fluid dynamics: III. The generalized streamline operator for multidimensional advection-diffusion systems, Comput. Methods Appl. Mech. Engrg. 58 (1986) 305-328.

[78] T.J.R. Hughes, L.P. Franca, I. Harari, M. Mallet, F. Shakib and T.E. Spelce, Finite element method for high-speed flows: Consistent calculation of boundary flux, AIAA-87-0556, AIAA 25th Aerospace Sciences Meeting, Reno, Nevada, 1987.

[79] T.J.R. Hughes, Recent progress in the development and understanding of SUPG methods with special reference to the compressible Euler and Navier-Stokes equations, Internat. J. Numer. Methods Fluids 7 (1987) 1261-1275.

[80] T.J.R. Hughes and L.P. Franca, A new finite element method for computational fluid dynamics: VII. The 
Stokes problem with various well-posed boundary conditions: Symmetric formulations that converge for all velocity/pressure spaces, Comput. Methods Appl. Mech. Engrg. 65 (1987) 85-96.

[81] T.J.R. Hughes and L.P. Franca, A mixed finite element formulation for Reissner-Mindlin plate theory: Uniform convergence of all high-order spaces, Comput. Methods Appl. Mech. Engrg. 67 (1988) 223-240.

[82] L.P. Franca and T.J.R. Hughes, Two classes of mixed finite element methods, Comput. Methods Appl. Mech. Engrg. 69 (1988) 89-129.

[83] A.F.D. Loula, L.P. Franca, T.J.R. Hughes and I. Miranda, Stability, convergence and accuracy of a new finite element method for the circular arch problem, Comput. Methods Appl. Mech. Engrg. 63 (1987) 281-303.

[84] A.F.D. Loula, T.J.R. Hughes, L.P. Franca and I. Miranda, Mixed Petrov-Galerkin methods for the Timoshenko beam problem, Comput. Methods Appl. Mech. Engrg. 63 (1987) 133-154.

[85] A.F.D. Loula, I. Miranda, T.J.R. Hughes and L.P. Franca, A successful mixed formulation for axisymmetric shell analysis employing discontinuous stress fields of the same order as the displacement field, in: Proceedings of the Fourth Brazilian Symposium on Piping and Pressure Vessels, Vol. 2 (Salvador, Brazil, 1987) 581-599.

[86] T.J.R. Hughes, M. Mallet and A. Mizukami, A new finite element formulation for computational fluid dynamics: II. Beyond SUPG, Comput. Methods Appl. Mech. Eng. 54 (1986) 341-355.

[87] T.J.R. Hughes and M. Mallet, A new finite element formulation for computational fluid dynamics: IV. A discontinuity-capturing operator for multidimensional advective-diffusive systems, Comput. Methods Appl. Mech. Engrg. 58 (1986) 329-336.

[88] C. Johnson and A. Szepessy, Shock-capturing streamline diffusion finite element methods for nonlinear conservation laws, in: T.J.R. Hughes and T.E. Tezduyar, eds., Recent Developments in Computational Fluid Dynamics, AMD Vol. 95 (ASME, New York, 1988) 101-108.

[89] E.G. Dutra do Carmo and A.C. Galeão, A consistent formulation of the finite element method to solve convective-diffusive transport problems, Rev. Brasileira Ciênc. Mec. 4 (1986) 309-340 (in Portuguese).

[90] A.C. Galeão and E.G. Dutra do Carmo, A consistent approximate upwind Petrov-Galerkin methods for convection-dominated problems, Comput. Methods Appl. Mech. Engrg. 68 (1988) 83-95.

[91] G.M. Hulbert, Space-time finite element methods for second-order hyperbolic equations, Ph.D. Thesis, Division of Applied Mechanics, Stanford University, Stanford, California, 1989.

[92] P.G. Ciarlet, The Finite Element Method for Elliptic Problems (North-Holland, Amsterdam, 1978). 